\title{
A Disturbance Observer Based Attitude Controller for Quadrotor Helicopters
}

\author{
Mengxuan Guo \\ School of Automation and Information Engineering \\ Xi'an University of Technology \\ Xi'an, China \\ 350538395@qq.com \\ Suoli Guo \\ Xi'an Research Institute of Hi-Tech \\ Xi'an, China \\ analog2k4@126.com
}

\author{
Xiaorong Wang \\ Early-childhood Teachers Normal College \\ Xi'an University of Arts and Science \\ Xi'an, China \\ xrongwang@126.com \\ Lining Tan \\ Xi'an Research Institute of Hi-Tech \\ Xi'an, China \\ oldbat@126.com
}

\begin{abstract}
This paper deals with the design of a robust attitude controller for a quadrotor helicopter subject to external disturbances. In order to attenuate these time varying and non-vanishing disturbances, we propose the use of a proportional integral observer to estimate and compensate for the disturbances. So, a two-stage procedure to design the robust attitude controller is proposed. First, a disturbance observer is designed. Then based on the observer, a backstepping controller is designed to stabilize the attitude of the quadrotor. Furthermore, the principle for selecting control parameters and stability analysis of the proposed composite controller are given. The observer output is used to compensate for the disturbance, and then the control law is generated by backstepping method. The observer based flight controller is able to provide robust attitude control of the quadrotor while relying only on knowledge of the bound of the disturbances. Simulations demonstrate the efficiency of the composite controller in the presence of external disturbances as well as model inaccuracy.
\end{abstract}

Keywords- Quadrotor helicopter; attitude control; proportional integral observer; backstepping control

\section{INTRODUCTION}

Quadrotor helicopter have been successfully used over the last few years. They have several advantages over traditional rotorcrafts in terms of mechanical complexity, maneuverability, flight safety and cost[1,2]. Nevertheless, quadrotor is an unstable system in nature and has a high nonlinear behavior[3,4]. Moreover, there usually exist various uncertainties in quadrotor control, which might cause disturbance torques and lead to an undesired motion of the aircraft. Therefore, an advance controller with high robustness has to be implemented for quadrotors' attitude control.

A wide variety of quadrotor attitude controller already exists in the literature. But few of them take into account external disturbances and model uncertainties. To achieve good performance in a hostile environment, a natural way is to estimate external disturbances and then compensate for them. In [5], a high gain observer and sliding mode controller for a quadrotor is designed. This controllerobserver is proved of good performance in simulations But the high gain feedback solutions have drawbacks that they may saturate actuators, excite high frequency modes etc. The work in [6] designed a sliding mode control driven by sliding mode disturbance observer (SMC-SMDO) based controller for quadrotor helicopter. The SMCSMDO allows for a continuous control robust to external disturbance and model uncertainties. But the parametric structure is so complex that the parameter tuning is difficult in this method.

In this paper, an alternative control strategy based on the proportional integral (PI) observer is developed for attitude stabilization of a quadrotor subject to external disturbances. The PI observer has a simple structure and its gain can be well controlled. This paper is organized as follows. The problem statement is given in section 2 . Section 3 presents the design of a PI disturbance observer. In Section 4, a composite controller based on the disturbance observer is designed. Numerical simulations are presented in Section 5. Finally presents the conclusions.

\section{PROBLEM STATEMENT}

The quadrotor has four rotors to generate thrust forces $T_{i}$ and rolling moments $M_{i}, \mathrm{i}=1,2,3,4$. Let $B=\left\{O_{b} x_{b} y_{b} z_{b}\right\}$ denotes the body fixed frame, whose origin is at the center of mass of the vehicle, and $E=\left\{O_{e} x_{e} y_{e} z_{e}\right\}$ denotes an inertial frame fixed with respect to the earth (see Fig .1). The attitude is represented by three Euler angles $\Theta=(\phi, \theta, \psi)^{T}$ and the body angular velocities by $\omega=(p, q, r)^{T}$. 
The quadrotor is considered to be a rigid body, whose dynamic equation of attitude motion is [7, 8]

$$
c_{\&}=-J^{-1} \omega \times J \omega+J^{-1} \tau+d
$$

where $\tau \in R^{3}$ is the input torques applied to the vehicle, $J \in R^{3 \times 3}$ is the known inertia matrix, and $d=\left(d_{x}, d_{y}, d_{z}\right)^{T}$ is the unknown external disturbances. Since disturbances are unknown and can not be measured, the major goals of this work are: a) design an observer to estimate the external disturbances and b) design $\tau$ to make $\omega$ stabilization with uniformly ultimate bounded error.

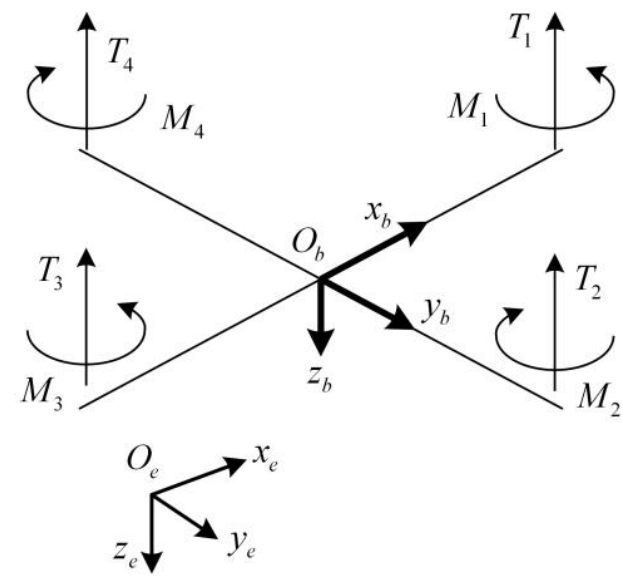

Figure 1. Quadrotor Helicopter Scheme

\section{DESIGN OF DiSTURBANCE OBSERVER}

It is well known that the PI observer is useful for linear systems with constant disturbance [9]. However, disturbances acted on a flying quadrotor depend on the state of aircraft and time. In general, there is no prior information about the disturbances, it is reasonable to make following assumption.

Assumption 1: The disturbance $d$ is bounded and satisfies

$$
\&=\zeta(t)
$$

where $\zeta(t)$ is an unknown function whose upper bound $\sup _{t \in[0, \infty)}\|\zeta(t)\| \leq b_{\zeta}$ and $b_{\zeta}$ is a positive constant.

This assumption implies that the disturbance varies in a bounded speed relative to the observer dynamics.

As shown in Fig .2, the dynamics of a PI observer for system (1) can be described by

$$
\left\{\begin{array}{l}
\&=-J^{-1} \omega \times J \omega+J^{-1} \tau+L_{1} \eta+\hat{d} \\
d=L_{2} \eta
\end{array}\right.
$$

where $L_{1} \in R^{3 \times 3}$ and $L_{2} \in R^{3 \times 3}$ are matrices designed latter, $\hat{\omega}=(\hat{p}, \hat{q}, \hat{r})^{T}$ is the estimate of angular velocities $\omega$ by observer and $\hat{d}=\left(\hat{d}_{x}, \hat{d}_{y}, \hat{d}_{z}\right)^{T}$ is the estimate of the disturbance $d, \eta$ is the estimation error of angular velocities $\omega$, i.e. $\eta=\omega-\hat{\omega}$. Then the problem is how to design the parametric matrices $L_{1}$ and $L_{2}$.

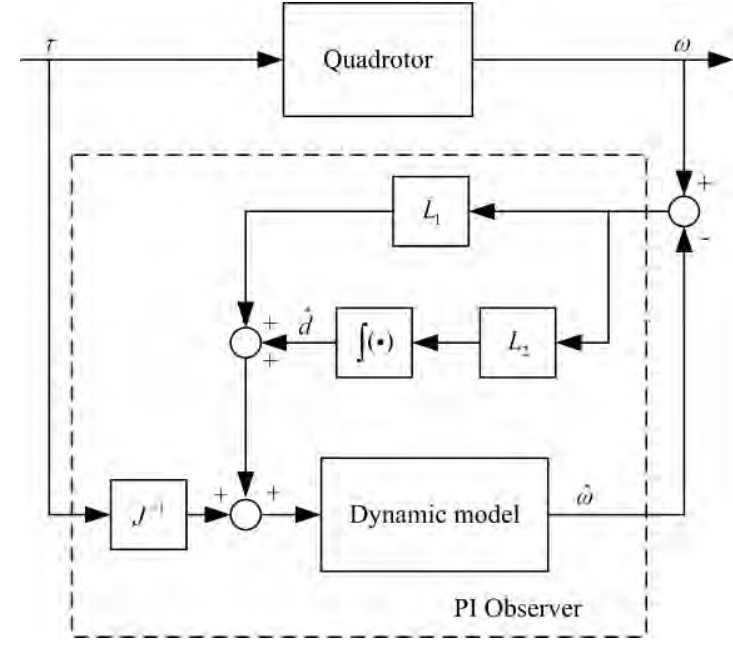

Figure 2. Structure of PI Observer

Define the estimation error of disturbance as $e=d-\hat{d}$. Then from (1) and (3), we have

$$
\left(\begin{array}{c}
\& \& \\
\&
\end{array}\right)=\left[\begin{array}{cc}
-L_{1} & I_{3} \\
-L_{2} & 0
\end{array}\right]\left(\begin{array}{l}
\eta \\
e
\end{array}\right)+\left(\begin{array}{c}
0 \\
I_{3}
\end{array}\right) \zeta=A\left(\begin{array}{l}
\eta \\
e
\end{array}\right)+B \zeta
$$

where $I_{n}$ denotes the identity matrix of dimension $n$.

From the dynamics (4) of the estimation errors it can be seen that, when $A$ is stable, the solution will be also bounded if $\zeta$ is bounded. Let us consider the characteristic polynomial of matrix. Therefore, when $L_{1}$ and $L_{2}$ are designed as $\begin{aligned} & L_{1}=k_{1} I_{3}, k_{1}>0 \\ & L_{2}=k_{2} I_{3}, k_{2}>0\end{aligned}$, the eigenvalues of $A-k_{1}$ and $-\frac{k_{1} k_{2}}{k_{2}+1}$ can be placed in the left-half complex plane. This means that the dynamics (4) are stable. Let

$$
\left[\begin{array}{l}
L_{1} \\
L_{2}
\end{array}\right]=\rho\left[\begin{array}{l}
L_{1}^{0} \\
\ell_{2}
\end{array}\right]
$$

with

$$
\rho>0 \text {. }
$$

Then (4) may be written as

$$
\frac{1}{\rho}\left(\begin{array}{c}
\& \\
\&
\end{array}\right)=\left[\begin{array}{cc}
-L_{1}^{0} & \frac{1}{\rho} I_{3} \\
-L_{2}^{0} & 0
\end{array}\right]\left(\begin{array}{l}
\eta \\
e
\end{array}\right)+\left(\begin{array}{c}
0 \\
\frac{1}{\rho} I_{3}
\end{array}\right) \zeta
$$

From (5) it follows that $\eta=0$ for $\rho \rightarrow \infty$. Then we get $e=0$ and can be seen that the estimates $\hat{\omega}$ and $\hat{d}$ of the PI observer (3) converge to the states $\omega$ and the external disturbance $d$ of the system (1) when $L_{1}$ and $L_{2}$ tend to infinity.

\section{DESIGN OF COMPOSITE CONTROLler}

The design procedure of the proposed composite controller consists of two steps. First, an original controller is designed by assuming the disturbance is measurable. Then, the composite controller is implemented by integrating the original controller with previous designed disturbance observer. During design of the original controller, backstepping technique is employed. 
Backstepping method is a recursive procedure for systematically selecting the control Lyapunov functions that allows the design of adaptive controllers for a class of nonlinear processes ${ }^{[11]}$.

\section{A. Original Controller design}

Define abbreviations and acronyms the first time they are used in the text, even after they have been defined in the abstract. Abbreviations such as IEEE, SI, MKS, CGS, $\mathrm{sc}, \mathrm{dc}$, and rms do not have to be defined. Do not use abbreviations in the title or heads unless they are unavoidable.

In order to design a backstepping controller for a quadrotor, we will rewrite its dynamic model (1) in statespace form $\mathbf{X}=f(\mathbf{X}, \mathbf{U})$ with inputs vector $U$ and states vector $X$ chosen as

$$
\begin{aligned}
& X=\left(x_{1}, x_{2}, x_{3}, x_{4}, x_{5}, x_{6}\right)^{T}=(\phi, \phi, \theta, \&, \psi, \psi \&)^{T} \\
& U=\left(u_{1}, u_{2}, u_{3}\right)^{T}=\left(\tau_{x}, \tau_{y}, \tau_{z}\right)^{T}
\end{aligned}
$$

Given desired roll angular $x_{1}^{d}$ and its measurement $x_{1}$, the tracking error is $z_{1}=x_{1}^{d}-x_{1}$. Consider the positive definite Lyapunov function as follow $V\left(z_{1}\right)=\frac{1}{2} z_{1}^{2}$. Then we use the Lyapunov theorem by considering the time derivative of $\mathrm{V}$ negative semi-definite $\&\left(z_{1}\right)=z_{1}\left(x_{2}-x_{2}\right)$. The stabilization of $z_{1}$ can be guaranteed by introducing a virtual control input $x_{2}^{d}: x_{2}^{d}=\alpha_{1} z_{1}+\alpha_{1}^{d}>0$. Then the time derivative of $\mathrm{V}$ is $\left.\& z_{1}\right)=-\alpha_{1} z_{1}^{2} \leq 0$. To make the roll angular velocity tracking the virtual control $x_{2}^{d}$, we repeat the above steps with tracking error between measured $x_{2}$ and the virtual control. Then the input $u_{1}$ is then designed to make $\left.\& z_{1}, z_{2}\right)<0$ :

$$
u_{1}=\left(I_{z z}-I_{y y}\right) x_{4} x_{6}+I_{x x}\left(\alpha_{2} z_{2}-\alpha_{1}^{2} z_{1}-d_{x}\right)
$$

with $\alpha_{1}>0$. The same steps are followed to obtain $u_{2}$ and $u_{3}$.

$$
\begin{aligned}
& u_{2}=\left(I_{x x}-I_{z z}\right) x_{2} x_{6}+I_{y y}\left(\alpha_{4} z_{4}-\alpha_{3}^{2} z_{3}-d_{y}\right) \\
& u_{3}=\left(I_{y y}-I_{x x}\right) x_{2} x_{4}+I_{z z}\left(\alpha_{6} z_{6}-\alpha_{5}^{2} z_{5}-d_{z}\right) \\
& \text { with } \alpha_{i}>0, i=3,4,5,6 .
\end{aligned}
$$

\section{B. Composite Controller design}

The observer based composite controller is obtained by replacing the disturbances in (8), (9) and (10) with its estimation yielded by the PI disturbance observer (4). Then we have

$$
\begin{aligned}
& u_{1}=\left(I_{z z}-I_{y y}\right) x_{4} x_{6}+I_{x x}\left(\alpha_{2} z_{2}-\alpha_{1}^{2} z_{1}-\hat{d}_{x}\right) \\
& u_{2}=\left(I_{x x}-I_{z z}\right) x_{2} x_{6}+I_{y y}\left(\alpha_{4} z_{4}-\alpha_{3}^{2} z_{3}-\hat{d}_{y}\right) \\
& u_{3}=\left(I_{y y}-I_{x x}\right) x_{2} x_{4}+I_{z z}\left(\alpha_{6} z_{6}-\alpha_{5}^{2} z_{5}-\hat{d}_{z}\right)
\end{aligned}
$$

To guarantee the stabilization of roll angular control subsystem, we design the parameter $\alpha_{2}$ in (8) as

$$
\alpha_{2}=\left\{\begin{array}{cc}
\frac{\beta_{1}}{\left|z_{2}\right|} & z_{2} \neq 0 \\
0 & z_{2}=0
\end{array}\right.
$$

where $\beta_{1}>b_{e, t_{e}}$.

Then the Lyapunov function becomes

$$
\&\left(z_{1}, z_{2}\right)=\left\{\begin{array}{cc}
z_{2}\left(-\beta-\left(d_{x}-\hat{d}_{x}\right)\right) & z_{2}>0 \\
0 & z_{2}=0 \\
z_{2}\left(\beta-\left(d_{x}-\hat{d}_{x}\right)\right) & z_{2}<0
\end{array}\right.
$$

When

$$
t>t_{e}
$$

Then

$$
\&\left(z_{1}, z_{2}\right)<\left\{\begin{array}{cc}
-2 z_{2} b_{e, t_{e}} & z_{2}>0 \\
0 & z_{2} \leq 0
\end{array} .\right.
$$

Since $b_{e, t_{e}}$ is a positive constant, we obtain $\left.\& \& z_{1}, z_{2}\right)<0$ when $t>t_{e}$. Similarly we can also design $\alpha_{4}$ and $\alpha_{6}$ for $u_{2}$ and $u_{3}$ to make the pitch and yaw control subsystem stable.

\section{Composite Controller Simulation}

In order to evaluate the proposed composite controller, simulations have been performed on the complete closed loop system in Matlab environment. External disturbances are assumed to be

$$
d=\left(\begin{array}{l}
d_{x} \\
d_{y} \\
d_{z}
\end{array}\right)=\left(\begin{array}{c}
0.5+\sin (\phi+\theta)+0.2 \sin (t) \\
0.5+\sin ((\phi+\theta) / 2)+0.2 \sin (2 t) \\
0.25
\end{array}\right)
$$

From (38) it can be seen that $d$ is time varying and nonvanishing. Especially, the disturbance torque acted on $b_{z}$ axis is constant and small due to the yaw angular of quadrotor aircraft is relatively hard to disturb.

The physical parameters for the dynamic model of the quadrotor are as in Table 1.

Table I: Physical parameters of the quadrotor
\begin{tabular}{|ccc|}
\hline Parameter & Value & Units \\
\hline$I_{x x}, I_{y y}$ & 0.177 & $\mathrm{~kg} / \mathrm{m}^{2}$ \\
\hline$I_{z z}$ & 0.334 & $\mathrm{~kg} / \mathrm{m}^{2}$ \\
\hline$C_{T}$ & 0.008 & \\
\hline$C_{M}$ & 0.0013 & \\
\hline $\boldsymbol{R}$ & 0.15 & $\mathrm{~m}$ \\
\hline $\boldsymbol{l}$ & 0.40 & $\mathrm{~m}$ \\
\hline
\end{tabular}

The initial states of the quadrotor and the PI observer are $X=(0.5,0.5,0.5,0.5,0,0)^{T}$ and $\hat{\omega}=(0,0,0)^{T}$, $\hat{d}=(0,0,0)^{T}$. The desired attitude is set to be $x_{1}^{d}=x_{3}^{d}=x_{5}^{d}=0$. Parameters of the composite controller are chosen to be $\alpha_{1}=\alpha_{3}=5, \alpha_{2}=\alpha_{4}=8, \alpha_{5}=4$ and $\alpha_{6}=6$. From Theorem 1, the gain parameters of PI observer is tuned at $k_{1}=10$ and $k_{2}=100$.

It should be noted that, in practice, the parameters of dynamic model in observer (7) is obtained by 
identification or empiricism, which implies that there must be some difference between the parameters in observer (7) and the flight dynamic model of the quadrotor. To take into account such inaccurate of parameters, the dynamic parameters in observer (7) are obtained as follow

$$
J_{\text {observer }}=J+J\left[\begin{array}{ccc}
\varepsilon_{1} & 0 & 0 \\
0 & \varepsilon_{2} & 0 \\
0 & 0 & \varepsilon_{3}
\end{array}\right]
$$

where $\varepsilon_{1,2,3}$ is uniformly distributed random variable on $[0.5,0.2]$.
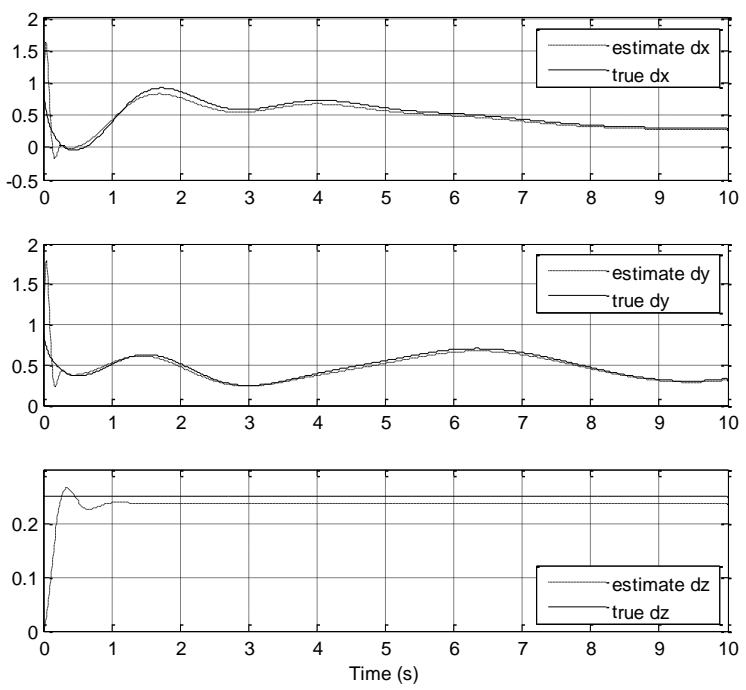

Figure 3. Estimate of the disturbance by the PI observer
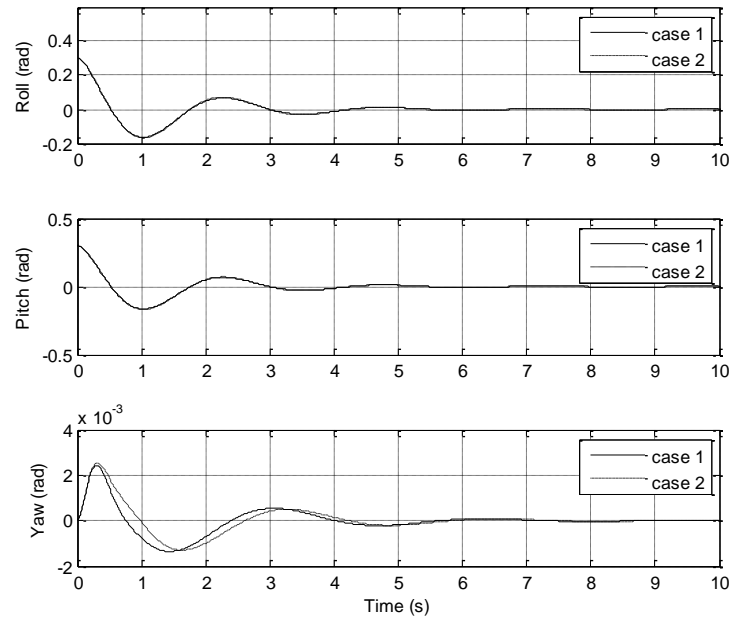

Figure 4. Attitude controlled by the composite controller

Simulations are carried out by using the noised parameters for the PI observer (4). Since the parameters in observer are noised, the estimate and estimation error of the disturbance by the PI observer are shown in Fig .3. It is clear that the estimate of disturbance is influenced by the parameters inaccuracy. This implies that the performance of the PI observer depends on the mathematical model of the plant. However, as the backstepping technique also has the ability of attenuating disturbances, the proposed composite controller is able to achieve good performance (see Fig .4). This proves the efficiency of the proposed control strategy.

\section{CONCLUSIONS}

In this paper, we have proposed a composite controller for attitude stabilization of a quadrotor helicopter in the presence of time varying disturbances. The proposed solution is based on a combin- ation of a PI observer and a backstepping controller. In the composite controller, the observer output is used to compensate for the disturbance, and then the control law is generated by backstepping method. Simulation results show the effectiveness of the proposed observer based control strategy.

\section{REFERENCES}

[1] S. Bouabdallah, P. Murrieri and R. Siegwart, "Design and control of an indoor micro quadrotor," in Proc. IEEE International Conference on Robotics and Automation, 2004, pp. 4393-4398.

[2] G. M. Hoffmann, H. Huang, S. L. Waslander and C. J. Tomlin, "Quadrotor helicopter flight dynamics and control: theory and experiment," in Proc. AIAA Guidance, Navigation, and Control Conference, Hilton Head, SC, August, 2007

[3] P. Pounds, R. Mahonyb and P. Corkec, "Modelling and control of a large quadrotor robot," Control Engineering Practice, vol. 18, no. 7, pp. 691-699, 2010.

[4] P. J. Bristeau, P. Martin, E. Salaun and N. Petit, "The role of propeller aerodynamics in the model of a quadrotor UAV," in Proc. European control conference, 2009, pp. 683-688.

[5] R. Zhang, Q. Quan and K. Y. Cai, "Attitude control of a quadrotor aircraft subject to a class of time varying disturbances," IET Control Theory and Applications, vol. 5, no. 9, pp. 1140-1146, 2011.

[6] L. Besnarda, Y. B. Shtesselb and B. Landruma, "Quadrotor vehicle control via sliding mode controller driven by sliding mode disturbance observer," Journal of the Frankin Institute, vol. 349, pp. 658-684, 2012.

[7] A. Tayebi and S. McGilvray, "Attitude stablilization of a VTOL quadrotor aircraft," IEEE Transactions on Control Systems Technology, vol. 14, no.3, pp. 562-571, 2006.

[8] S. Bouabdallah and R. Siegwart, "Backstepping and sliding-mode techniques applied to an indoor micro quadrotor," in Proc. IEEE International Conference on Robotics and Automation, 2005, pp. 2247-2252.

[9] S. Beale and B. Shafai, "Robust control design with a proportional integral observer," International Journal of Control, vol. 50, no. 1, pp. 97-111, 1989.

[10] I. Fantoni and R. Lozano, Nonlinear control for underactuated mechanical systems, Springer, 2002

[11] P. Pounds, R. Mahony and P. Corke, "System identification and control of an aerobot drive system", in Proc. Information, Decision and Control, 2007, pp. 154-159.

[12] K. M. Zemalache, L. Beji and H. Maaref, "Two inertial models of $\mathrm{x} 4$-flyers dynamics, motion planning and control," Integrated Computer-Aided Engineering, vol. 14, no. 2, pp. 107-119, 2007.

[13] N. Guenard, T. Hamel and R. Mahony, "A practical visual servo control for an unmanned aerial vehicle," IEEE Transactions on Robotics, vol. 24, no. 2, pp. 331-340, 2008.

[14] T. Madani and A. Benallegue, "Sliding mode observer and backstepping control for a quadrotor unmanned aerial vehicles," in Proc. American control conference, NY, 2007, pp. 5887-5892. 